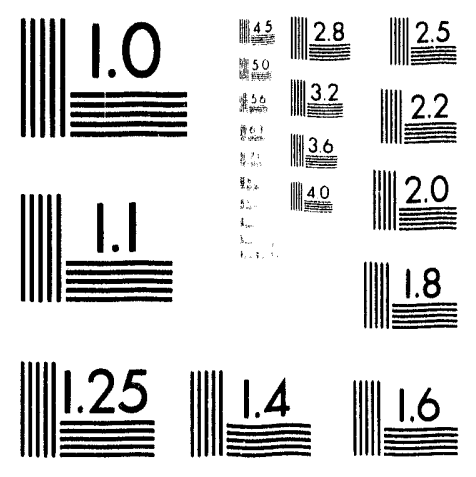



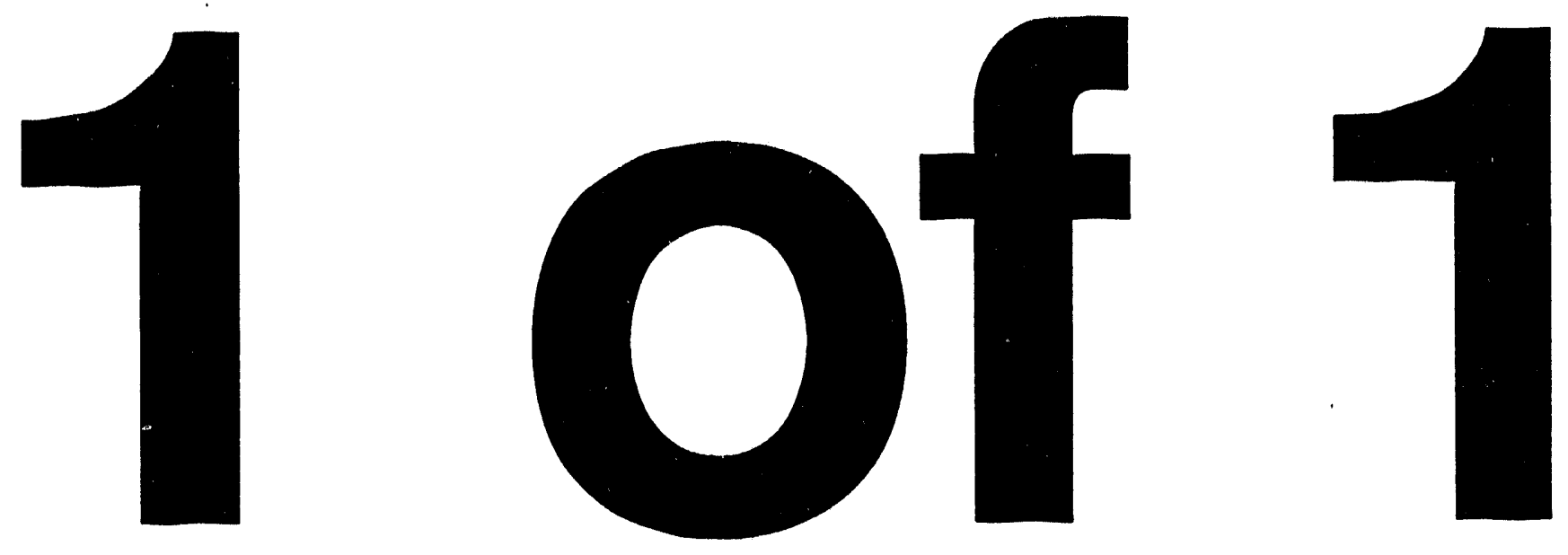


\title{
The Use of Harmonics in 3-D Magnetic Fields
}

\author{
S. Caspi, M. Helm, LJ. Laslett \\ Lawrence Berkeley Laboratory \\ University of California \\ Berkeley, CA 94720
}

\begin{abstract}
Motivated by the need for new means for specification and determination of 3-D fields that are produced by electromagnetlc lens elements in the region interior to coll windings and seeking to obtain techniques that will be convenient for accurate conductor placement and dyanmical study of particle motion, we have generallized the representation of a 2-D magnetic field to 3-D. We have shown that the 3-D magnetlc field components of a multipole magnet In the curl-free divergence-free reglon near the axis $r=0$ can be derived from one dimensional functlons $A_{n}(x)$ and their derivatives.
\end{abstract}

\section{INTRODUCTION}

The performance quality of beam-transport lines and of particle accelerators is basically dependent upon the characteristics of the magnetic fields that normally are employed to guide and focus the beam. We propose here a form that expresses a general 3-D magnetic field and hope that the proposed form will prove to constitute an efficient means for transferring field information and will prove convenient for future dynamical computations. We shall also be concerned in the present note with the object of giving adequate attention to the influence of end-winding configurations.

In the region interior to coil windings of accelerator magnets the three spatial components of magnet fields can be expressed in terms of "harmonic components" proportional to functions $\sin (n \theta)$ or $\cos (n \theta)$ of the aximuthal angle. The $r, z$ dependence of any such component can then be expressed in terms of powers of $r$ times functions $A_{n}(z)$ and their derivatives. For 2-D configurations $\mathrm{Bz}$ of course is identically zero, the derivatives of $A_{n}(z)$ vanish, and the harmonic components of the transverse field then acquire a simple proportionality $B_{r, n} \propto r^{n-1} \sin (n \theta), B_{\theta, n^{\propto}} \propto r^{n-1} \cos (n \theta)$ [for the non-skew configurations], whereas in a 3-D configuration the more complex nature of the field gives rise to additional socalled "pseudo-multipole" components as judged by additional powers of $r$ required in the development of the field.

Manuscript received September 20, 1993. This work was supported by the Director, Office of Energy Research, Office of High Energy and Nuclear Physics, High Energy Physics Division, U.S. Department of Energy, under Contract Na. DE-ACO3-76SF00098.
Computation of the 3-D magnetic field arising at a sequence of field points, as a direct result of a specified current configuration or coil geometry, can be calculated explicitly through use of the Biot-Savart law and from such data the coefficients can then be derived for a general development of the type indicated above. We indicate, discuss, and illustrate two means by which this development may be performed, employing in the second of these methods computations based on the use of Differential Algebra (DA). In this report we apply both methods to the "end" region of a $40 \mathrm{~mm}$ bore SSC quadrupole; calculating first the field harmonics and then reconstructing the field comparing both results with direct Biot-Savarlicalculation [1], [2].

\section{3-D FIELD REPRESENTATION}

We note that in the curl-fres divergence-frce region near the axis $r=0$ the field components may be expressed as given by $\vec{B}=-\nabla V$ where $V$ is a scalar potential function for which $\nabla^{2} \mathrm{~V}=0$. The proposed general solution for the scalar potential of the 3-D magnetic field including both "skew" and "non-skew" terms of all integer harmonic of order. $n$ (including $n=0$ ) can be written in the form: .

$$
\begin{aligned}
V=-\sum_{n=0} r^{n} \sum_{k=0} & \frac{(-1)^{k+1} n !}{2^{2 k} k !(n+k) !} r^{2 k} \\
& {\left[A_{n}^{(2 k)}(z) \sin n \theta-\tilde{A}_{n}^{(2 k)}(z) \cos n \theta\right] }
\end{aligned}
$$

(the negative sign in front of the "skew" term complies with' the right hand rule).

The magnetic field can be derived accordingly as:

$$
\begin{aligned}
& B_{r}=-\frac{\partial V}{\partial r}=\sum_{n}\left[\dot{g}_{m} r^{n-1} \sin n \theta-\bar{g}_{r n} r^{n-1} \cos n \theta\right] \\
& B_{\theta}=-\frac{n}{r} V=\sum_{n}\left[g_{\theta n} r^{n-1} \cos n \theta+\bar{g}_{\theta n} r^{n-1} \sin n \theta\right] \\
& B_{z}=-\frac{\partial V}{\partial z}=\sum_{n}\left[g_{z n} r^{n} \sin n \theta-\bar{g}_{z n} r^{n} \cos n \theta\right]
\end{aligned}
$$

where the $g$ 's are general functions of $r$ and $z$ that include the appropriate "non-skew" and "skew" terms $A_{n}(z)$ and $\bar{A}_{n}(z)$. In order that the series satisfy the differential equation we express the coefficients $\mathrm{grn}, \mathrm{g} \theta \mathrm{n}, \mathrm{gzn}$ as general functions of $r$ and $z$ as shown below for the "non-skew" series:

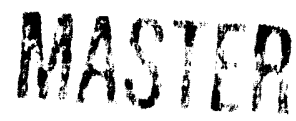




$$
\begin{aligned}
& g_{r n}(r, z)=\sum_{k=0}(-1)^{k+1} \frac{n !(n+2 k)}{2^{2 k} k !(n+k) !} A_{n}^{(2 k)}(z) r^{2 k} \\
& g_{\theta n}(r, z)=\sum_{k=0}(-1)^{k+1} \frac{n ! n}{2^{2 k} k !(n+k) !} A_{n}^{(2 k)}(z) r^{2 k} \\
& g_{z n}(r, z)=\sum_{k=0}(-1)^{k+1} \frac{n !}{2^{2 k} k !(n+k) !} A_{n}^{(2 k+1) r 2 k}
\end{aligned}
$$

where $A_{a}^{(2 k)}$ are the (2k) derivatives with respect to $\mathrm{z}$.

For the "skew" terms replace $A_{n}^{(2 k)}$ with $\bar{A}_{n}^{(2 k)}$.

We bave computed $g \mathrm{~m}, 8 \theta n, g \mathrm{~m}$ from which we calculated $A_{n}(2)$ and derivatives. In general the need for high derivatives and their relative contribution to the magnetic field should be considered only when field values are needed close to the winding.

The form proposed above in which a 3-D field throughout an interior region is expressed in terms of the function $A_{n}(z)$ and its derivatives may appear analogous in spirit to the similar description of fields with rotational symmetry in terms of a z-directed field (solenoid) and its derivatives along the symmetry axis.

The representation specified above for 3-D magnetic fields, written in terms of functions $A_{n}(z)$ and $\bar{A}_{n}(z)$ and their derivatives, will describe a field that formally is both divergence free and curl free provided that the summations are not truncated. If, however, we wish to truncate these series expressions, we at best can only do so in such a way that one, but not both, of these conditions is satisfied. Thus, if we wish to preserve the divergence condition $\nabla \overrightarrow{\mathrm{B}}=0$ (so that the consequent dynamical equations for charged-particle motion can be derived from a Hamiltonian function in which the magnetic field is described by a vector potential), we sbould take care that the sum over the index $k$ (Equation 3 ) in the series for $B_{2}$ should terminate at a value of $k$ that is less by unity than the termination value for this index in the series for the transverse field components $\mathrm{B}_{\mathrm{r}}$ and $\mathrm{B}_{\boldsymbol{\theta}}$.

We have developed two methods for computing the functions $A_{n}(z)$ and their derivatives and used them to calculate the magnetic field anywhere inside the curl-free divergence-free region.

\section{A. A Finite-Difference Method}

The first method is using Biot-Savart to compute the magnetic field from a set of "line current" segments that simulate the coil windings. Calculating the magnetic field components at several discrete angles on an arc (e.g. at a 50\% radius of the inner bore) so chosen that a Fourier analysis can conveniently be performed and the harmonic components evaluated and stored for successive values of $z$. Next these components are numerically differentiated with respect to $z$ to provide $A$ values and their derivatives up through the 6 th order.

We estimate the function of $A\left(z_{0}\right)$ through the use of values of $g_{r}\left(z_{0}\right)$ and $g_{\theta}\left(z_{0}\right)$ and the values of $g_{z}\left(z_{0} \pm 3 h\right)$, $g_{z}\left(z_{0} \pm 2 h\right), g_{z}\left(z_{0} \pm h\right)$ and $g_{z}\left(z_{0}\right)$, and we presume that $a$ Taylor-series development in this neighborhood can ignore derivatives of order 7 and greater. As shown [2], we can obtain values of the odd derivatives $A^{\prime}, A^{\prime \prime \prime}$ and $A^{V}$ that permit one to evaluate the even derivatives at $z_{0}$ with similar accuracy. Finally to obtain the function $A\left(z_{0}\right)$ itself we employ a suitable average of the quantities $g_{r}\left(z_{0}\right)$ and $g_{\theta}\left(z_{0}\right)$.

The numerical quality of the As was proven to be of high accuracy especially in the vicinity of the radius at which the Fourier analysis was performed. This is not a surprise however, but it does restrict somewhat the region of interpolation. On the other hand this method provides the means for calculating fields closer to the windings by choosing a proper radius. It should be noted that this method is independent of which method is used in calculating the magnetic field and therefore can be applied to problems with iron. If the iron is allowed to saturate the As become a function of the current and therefore more complicated.

\section{B. Differential Algebra Method}

It appiars possible in principle to proceed directly, through use of the Differential Algebra program developed by M. Berz [3], from a formula representing a Biot-Savart evaluation of $B_{y}\left(B_{\theta}\right.$ at $\left.\theta=0\right)$ to evaluation of the functions $A_{n}(k)(z)$ that may serve to describe the field in the manner shown by Equations (2)-(3). In particular one may by this means (i) avoid the need to make an initial preliminary Fourier analysis of the field components and (ii) avoid the need to approximate various derivatives of functions $A_{n}(z)$ through use of finite difference forms applied to field components at some non-zero radius. It should be noted that when the magnetic field can be expressed in.terms of analytical functions of $z$ the $A_{n}(z)$ 's and their derivatives can be computed directly as in the simple case where $A_{n}(z)=\cos \left(\frac{\pi z}{L}\right)$ and the solution is separable and reduces to Bessel functions in $r$.

The best part of using DA is that higher derivatives can be calculated to any desired order (limited by truncation errors) and a degree of simplification introduced in the fact that a Fourier analysis is not needed. The major drawback is the computation time which is dominated by the DA package and is slow for the problem size used here. In addition we had to make several modification to the Biot-Savart formula to 
reduce errors. Normalizing each line current to its length was proven very effective especially when long segments are present (e.g. a long straight section).

\section{Magnetic Field in the SSC Quad End.}

We have applied the methods previously described to the end region of the $40 \mathrm{~mm}$ bore SSC quadrupole and generated the first four allowed harmonic function $A 2,6,10,14$ with their corresponding pseudo harmonics. As many as 6 pseudo harmonic functions were calculated using the finite difference method and as many as 16 such functions were calculated with the DA method with no obvious loss of numerical stability. The end region schematically shown in Fig. 1 was used in calculating the $A$ 's, however during computation each turn was replaced with a 3 dimensional multistrand cable configuration. The result of the A's are shown in Fig. 2 for the quadrupole, dodecapole and 10 pole. The iron contribution was ignored in this part in order to make a direct comparison between both methods.

With the A's computed we have reconstructed the 3-D field components along $z$ at $a$ given $r$ and $\theta=45$ using Equation 2 . The transverse field component $B_{r}\left(B_{\theta}=0\right)$ and $B_{Z}$ are ploted in Fig. 3 for $r=1.0 \mathrm{~cm}$. We have also computed the field along the same path using the Biot-Savart formula directly and compared it with both methods. The corresponding difference between both methods and Biot-Savart are plotted in Fig. 4. The process was repeated at $r=25 \%, 50 \%, 75 \%$, 85\% and $95 \%$ of the physical bore and the maximum difference plotted as a function of the reduced radius in Fig. 5 .
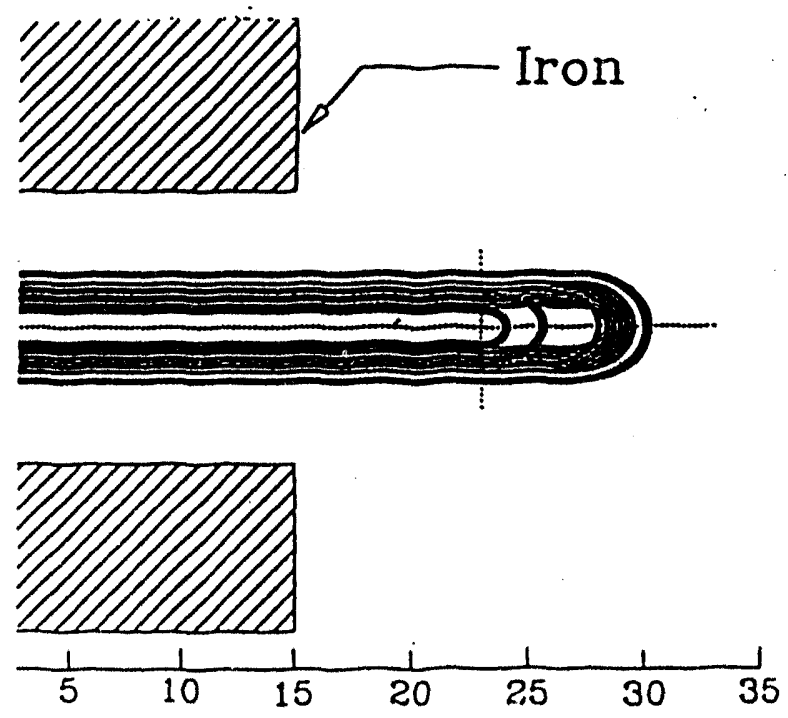

Fig. 1. The two layer coil and iron configurmion in the end region of the SSC quadrupole magnet.


Fig. 2. Harmonics and the first three pseudo harmonics associated with $A_{2}$. A $_{6}$ and $A_{10}$ 

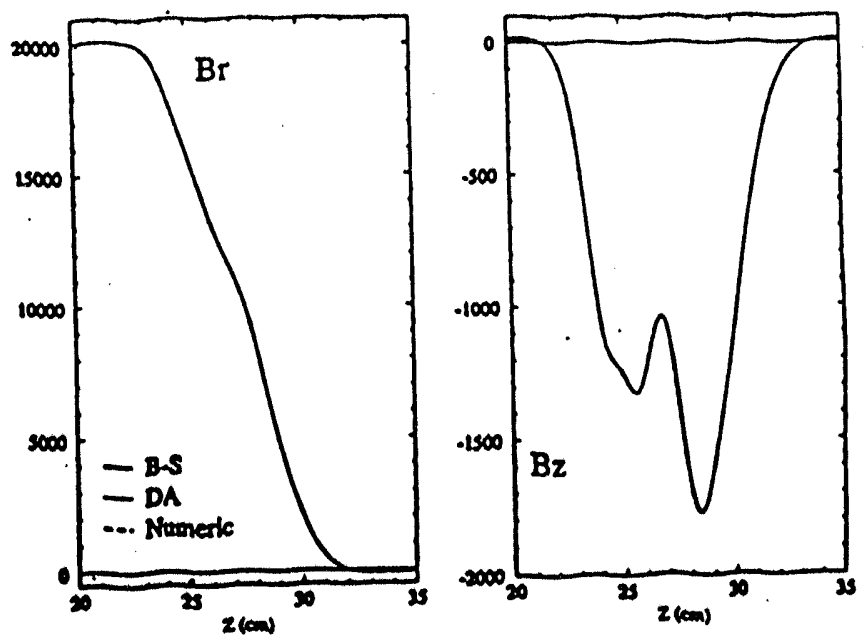

Fig. 3. Redial and axial field along the end region ( $r=1.0 \mathrm{~cm}, \theta=45$ ). usiag be two methods and Bio-Savart.
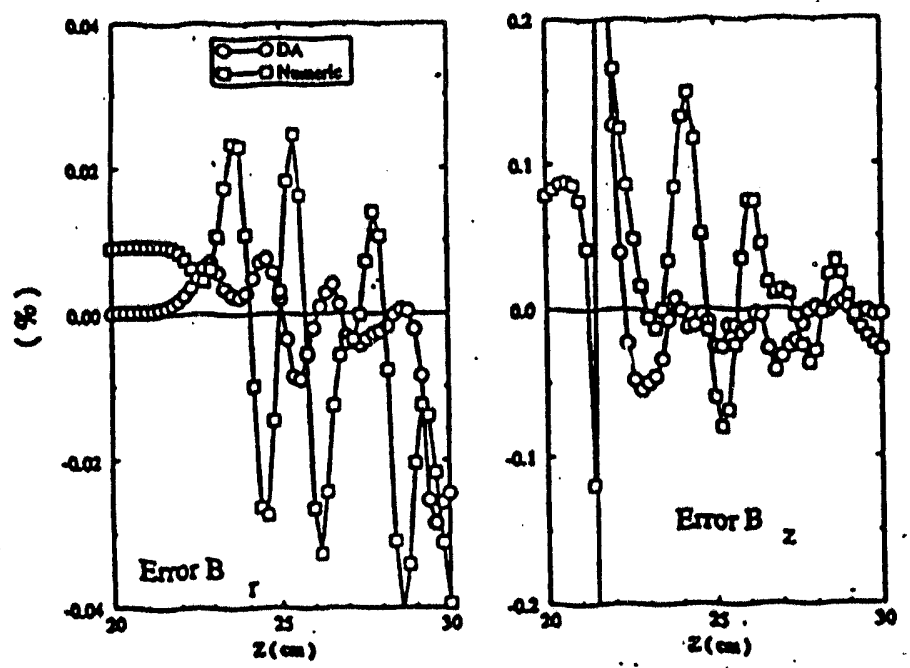

Fis. 4. Errors aseociuted with both methods ia prediating the fiold an $=1.0 \mathrm{~cm}, \theta=45$.

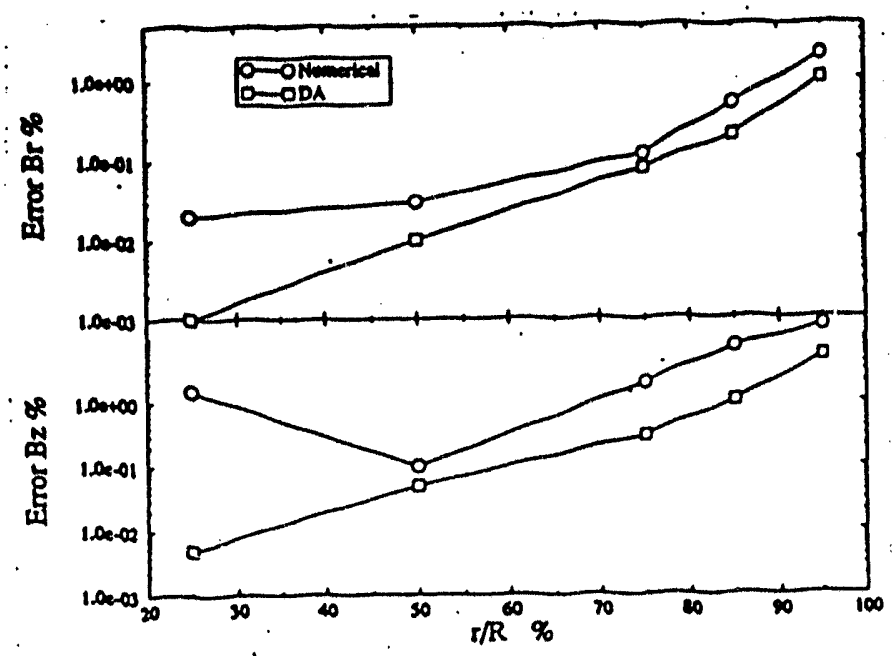

Fig. 5. Maximum error in field as a function of the reduced bore radius.

\section{CONCLUSION}

We have demonstrated a method of reducing magnetic field calculation of accelerator magnets to a finite set of harmonic functions which can subsequently be used to reconstruct the field anywhere within the physical bore. The method was demonstrated on the end region of a quadrupole magnet and the results compared with direct computation. The quality of the results suggests that a truncated series of harmonic functions is sufficient to accurately predict the magnetic field components inside the physical bore. We also point out that the advantage of the finite difference method is its speed and ability to include iron contributions. The DA method on the other hand although slower seems to give a more accurate prediction of field.

\section{Acknowledgment}

Earlier this year, Dr. Jackson Laslett died after a short illness. We have worked closely with Jackson for the past 9 years and were privileged in benefiting from his insight into physics and computational science. "Jackson is amazing" was the most heard of quote from many of the people who knew him. We couldn't agree more. It was a pleasure having the opportunity to work with Jackson, a man of outstanding talent and a true gentleman. We shall miss him.

\section{References}

[1] S. Caspi, M. Helm, and LJ. Laslett, "3D Field Harmonics", Lawrence Berkeley Lab. Report, LBL-30313. SC-MAG 328, March 1991.

[2] S. Caspi, M. Helm, LJ. Laslett and V.O. Brady, “An Approach to 3D Magnetic Field Calculation Using Numerical and Differential Algebra Methods", Lawrence Berkeley Lab. Report, LBL-32624, SC-MAG 395, July 1992.

[3] M. Berz, "Differential Algebra - A New Tool", LEEE Particle Accelerator Conference, vol. 3, pp. 1419-1423. March 1989. 

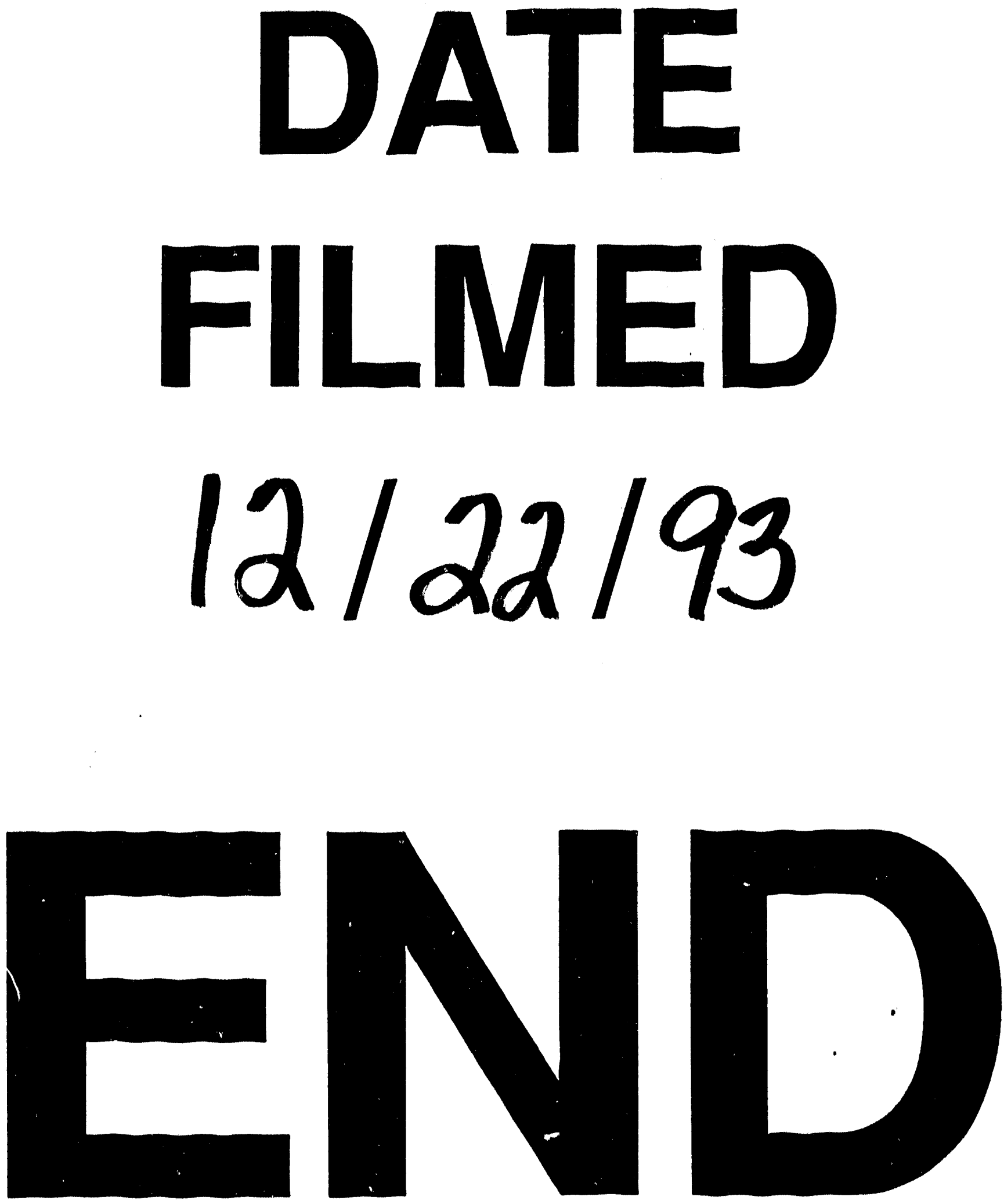
